\title{
Deep semi-supervised learning for automatic segmentation of inferior alveolar nerve using a convolutional neural network
}

\author{
Ho-Kyung Lim ${ }^{1 \dagger}$, Seok-Ki Jung ${ }^{2 \dagger}$, Seung-Hyun Kim ${ }^{3}$, Yongwon Cho ${ }^{4^{*}}$ and In-Seok Song ${ }^{5^{*}}$
}

\begin{abstract}
Background: The inferior alveolar nerve (IAN) innervates and regulates the sensation of the mandibular teeth and lower lip. The position of the IAN should be monitored prior to surgery. Therefore, a study using artificial intelligence (AI) was planned to image and track the position of the IAN automatically for a quicker and safer surgery.

Methods: A total of 138 cone-beam computed tomography datasets (Internal: 98, External: 40) collected from multiple centers (three hospitals) were used in the study. A customized 3D nnU-Net was used for image segmentation. Active learning, which consists of three steps, was carried out in iterations for 83 datasets with cumulative additions after each step. Subsequently, the accuracy of the model for IAN segmentation was evaluated using the 50 datasets. The accuracy by deriving the dice similarity coefficient (DSC) value and the segmentation time for each learning step were compared. In addition, visual scoring was considered to comparatively evaluate the manual and automatic segmentation.
\end{abstract}

Results: After learning, the DSC gradually increased to $0.48 \pm 0.11$ to $0.50 \pm 0.11$, and $0.58 \pm 0.08$. The DSC for the external dataset was $0.49 \pm 0.12$. The times required for segmentation were 124.8, 143.4, and $86.4 \mathrm{~s}$, showing a large decrease at the final stage. In visual scoring, the accuracy of manual segmentation was found to be higher than that of automatic segmentation.

Conclusions: The deep active learning framework can serve as a fast, accurate, and robust clinical tool for demarcating IAN location.

Keywords: Deep learning, Convolutional neural network, Automatic segmentation, Inferior alveolar nerve

\section{Background}

The inferior alveolar nerve (IAN) is a branch of the mandibular division of the trigeminal nerve, and it is an important anatomical structure in dentistry since it transmits the sensation in mandibular teeth and lower

\footnotetext{
*Correspondence: dragon1won@korea.ac.kr; densis@korea.ac.kr ${ }^{\dagger}$ Ho-Kyung Lim and Seok-Ki Jung have contributed equally to this work ${ }^{4}$ Department of Radiology and Al Center, Korea University College of Medicine, Korea University Anam Hospital, 73, Goryeodae-ro, Seongbuk-gu, Seoul 02841, Republic of Korea

${ }^{5}$ Department of Oral and Maxillofacial Surgery, Korea University Anam Hospital, 73, Goryeodae-ro, Seongbuk-gu, Seoul 02841, Republic of Korea Full list of author information is available at the end of the article
}

lip [1]. The location of the IAN influences the prognosis of an orthognathic surgery, pathological treatment of benign, or malignant, and outpatient procedures, such as dental implants and extraction [1-3]. Compared to plain radiography, cone-beam computed tomography $(\mathrm{CBCT})$ can easily determine the position of the IAN. However, it is still difficult to intuitively judge the three-dimensional (3D) position, and an operator is required to determine the location. Additionally, even if the location is judged, under static IAN tracking, the possibility of nerve damage still exists [4]. 
Since artificial intelligence (AI) was first introduced, the deep learning technology has been advanced consistently $[5,6]$. Deep learning technology using a convolutional neural network $(\mathrm{CNN})$ exhibits excellent performance in image analysis. This involves object detection, which refers to finding a specific object in an image; object classification, which refers to classifying the corresponding object; and object segmentation, which refers to finding and separating the area of a specific object [7]. Presently, AI can analyze and evaluate images, which can contribute significantly in medical diagnosis $[8,9]$.

Therefore, the segmentation technique has been studied to determine the precise position of the IAN using deep learning technology and $3 \mathrm{D}$ CBCT data. If the position of the IAN is automatically detected on a CBCT image, the IAN can be dynamically tracked during the surgery, which reduces the burden on the surgeon and improves the accuracy of the procedure while making it quicker and safer.

Earlier, the statistical shape model (SSM) described by Abdolali et al. was used for automatic segmentation of the IAN [10]. However, the SSM approach requires annotations for the segmented mandible during training. Thus, additional manual annotation or algorithm development is required. In another method described by Moris et al., segmentation was performed by selecting a predefined threshold for the grayscale value of the image that best separates the IAN from other tissues [11]. However, since the Hounsfield unit (HU) scale is not accurate in CBCT scans and the results depend on the quality of the imaging device, methodological limitations exist [12].

In the present study, a stepped 3D nnU-Net method that enables active learning was utilized to increase training efficiency with limited data and reduce labeling efforts by including AI [13]. Here, a person evaluates and corrects the result obtained from an $\mathrm{AI}$, and the $\mathrm{AI}$ learns iteratively for organic and dynamic performance improvement. In order to explore the possibility of dynamic tracking of IAN, our study aimed at determining the precise position of the IAN using AI and evaluated its accuracy by comparing the predicted position of the nerve to the position manually designated by multiple specialists. In addition, it was verified that the segmentation accuracy and annotation efficiency can be improved with active learning.

\section{Material and methods \\ Dataset collection}

A study using dataset of multi-center was conceived and conducted for the study. Data pertaining to 138 patients who visited Korea University Anam Hospital (A), Korea University Ansan Hospital (B), and Korea University Guro Hospital (C) between January 2018 and May 2020 were utilized in this study [Internal: 98 patients (A), External: 40 patients (20 each from B and C)]. Only CBCT data of adults whose mandible and inferior alveolar nerves have matured were extracted. In order to avoid the effects of marrow dystrophy, data of those aged 60 years or older were not collected. The equipment used were KAVO 3D Exam 17-19 (Imaging Sciences International, Hatfield, PA, USA) at A, CS 9300 (Carestream Dental, GA, USA) at B, and Vatech Pax-Reve3D (Vatech, Seong-Nam, Gyeonggi-do, Korea) at C (Table 1). This study was conducted in accordance with the Declaration of Helsinki under the approval of the institutional ethics committee of each hospital [approved by the Institutional Review Board of A (2020AN0410, 11/01/2021), B (2021AS0041, 09/02/2021), and C (2021GR0148, 07/04/2021)].

Table 1 Characteristics and data collection parameters for the study population

\begin{tabular}{|c|c|c|c|c|}
\hline Characteristic & $\begin{array}{l}\text { Training and tuning } \\
(\mathrm{A}) \\
(\mathrm{N}=83)\end{array}$ & $\begin{array}{l}\text { Internal validation } \\
(\mathrm{A}) \\
(\mathrm{N}=15)\end{array}$ & $\begin{array}{l}\text { External validation } \\
(\mathrm{B}) \\
(\mathrm{N}=20)\end{array}$ & $\begin{array}{l}\text { External validation } \\
(\mathrm{C}) \\
(\mathrm{N}=20)\end{array}$ \\
\hline Age (in years) & $59.9 \pm 17.2$ & $63.1 \pm 16.9$ & $40.0 \pm 19.7$ & $43 \pm 18.6$ \\
\hline Male & 44 & 8 & 10 & 10 \\
\hline Female & 39 & 7 & 10 & 10 \\
\hline Tube voltage (kV) & 120 & 120 & 90 & 90 \\
\hline Tube current (mA) & 5 & 5 & 4 & 4 \\
\hline Scan time (s) & 16.8 & 16.8 & 14.3 & 15 \\
\hline Voxel size (mm) & 0.3 & 0.3 & 0.3 & $0.08 \sim 0.25$ \\
\hline $\mathrm{FOV}\left(\mathrm{mm}^{2}\right)$ & $230 \times 170$ & $230 \times 170$ & $170 \times 135$ & $150 \times 150$ \\
\hline Focal spot (mm) & 0.58 & 0.58 & 0.70 & 0.50 \\
\hline
\end{tabular}

Internal dataset: Korea University Anam Hospital (A); External dataset: Korea University Ansan Hospital (B) and Korea University Guro Hospital (C) FOV, field-of-view 


\section{Training architecture}

The overall design of the study was similar to a previous one where lesion and air of the maxillary sinus were segmented [14]. In the present study, a customized nnU-Net was used for image segmentation [13] (Fig. 1). The nnUNet structure consists of an encoder that uses a convolutional filter and max pooling, as well as a decoder that decodes the signal back to the original dimension. The first layer of the encoder consists of 30 convolutional filters, wherein the spatial information is compressed and reduced. Further, the spatial information is restored using deconvolution by the decoder, and the information on the boundary is reinforced.

\section{Active learning}

The active learning process comprised three steps. Here, a new dataset was added at each step while using the model learned in the previous step. This improved the model's performance as much as possible with a limited training dataset.

The internal dataset of 98 patients was randomly divided into training, tuning, and testing sets at a ratio of 7:1:2 (68 patients: 15 patients: 15 patients).
Two-dimensional (2D) segmentation maps along the vertical axis of the CBCT images were constructed. The ground truth of the IAN was provided by three specialists. The overall segmentation was performed using AVIEW Modeler software (version 1.0.3, Coreline Software, Seoul, Korea). All input volumes were resized to $320 \times 320$ pixels, and the intensity was normalized. Data augmentation was performed using gamma adjustment, random scaling, random rotation, mirroring, and random elastic deformations. The dilation limit of the IAN thickness was set to $3 \mathrm{~mm}$.

In the first stage, the ground truth was established by three specialists for 19 CBCT datasets from A. The AVIEW Modeler software was contained with the function of labeling the IAN in 3D view. First, the IAN was segmented automatically in the program, and then the IAN position was corrected manually on the sagittal, coronal, and axial cross-sectional images. After the labeling process, training was conducted to segment the IAN using the labeled dataset. After the first step, a new training process was conducted with 49 datasets that added 30 new unlabeled datasets. Using the model learned in the process, segmentation was first performed, and then

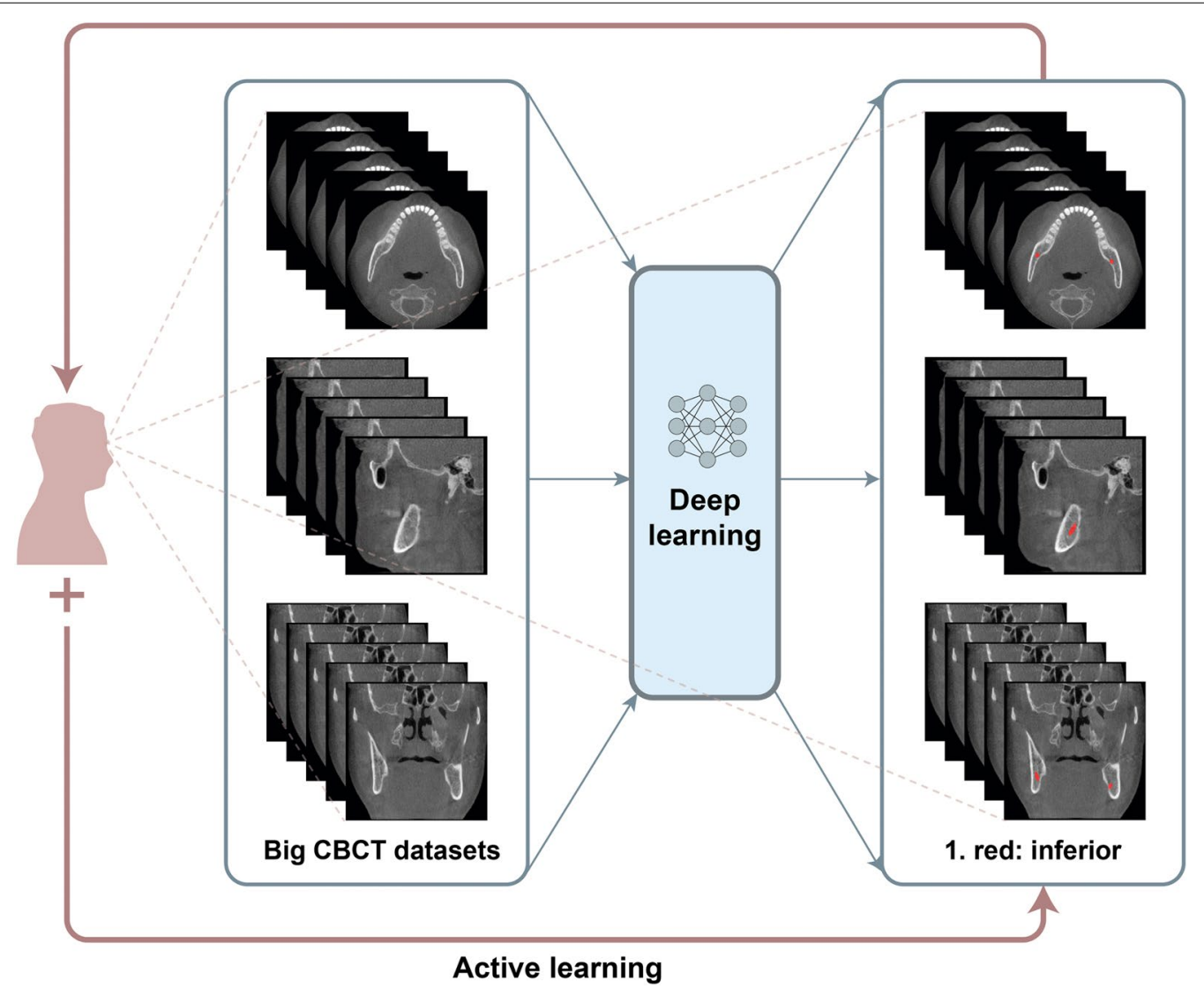

Fig. 1 Deep learning architecture of the customized 3D U-Net adapted from nnU-Net 
three specialists adjusted it to determine the ground truth. In the third step, 34 new unlabeled datasets were added again, and additional training was performed using a total of 83 datasets. This process was repeated to train the model and improve its performance. Moreover, the model was tested using the remaining dataset. The accuracy of each model in IAN segmentation considering a total of 55 datasets, including the remaining 15 internal scans (A) and 40 external scans (20 each from B and C) were evaluated (Fig. 2).

\section{Training setup}

The random rectified linear unit (ReLU) function as the activation function was used. The cross-entropy, dice coefficient, and boundary loss functions were used for training. For IAN segmentation learning, adaptive layerinstance normalization (AdaLin) and Adam optimizer were used along with an initial learning rate of $3 \times 10^{-4}$, and a 12-weight decay value of $3 \times 10^{-5}$. The change in the learning rate was reduced by a factor of 0.2 if the loss value did not improve during 30 epochs. Learning was terminated after 1000 epochs or when the learning rate was below
$10^{-6}$. For optimal model selection, tuning was performed throughout the training process per an epoch using the tuning set among training set in Table 1 . The deep learning model was constructed using the TensorFlow framework (1.15.0). Learning was performed using an NVIDIA Titan RTX graphics card of $24 \mathrm{~GB}$. The batch size was set to six, and the learning procedure was completed in approximately 100 epochs.

\section{Evaluation}

Segmentation was evaluated using dice similarity coefficient (DSC) values. DSC comparatively analyzed the ground truth and the predicted values. It was obtained by determining the ratio of the overlapped area between the predicted value and the ground truth with the sum of the predicted value and the ground truth. A value closer to 1 indicated the accuracy of the method. DSC was calculated as follows:

$$
\operatorname{DSC}\left(V_{s e g}, V_{g s}\right)=\frac{2\left|V_{\text {seg }} \cap V_{g s}\right|}{\left|V_{\text {seg }}\right|+\left|V_{g s}\right|},
$$

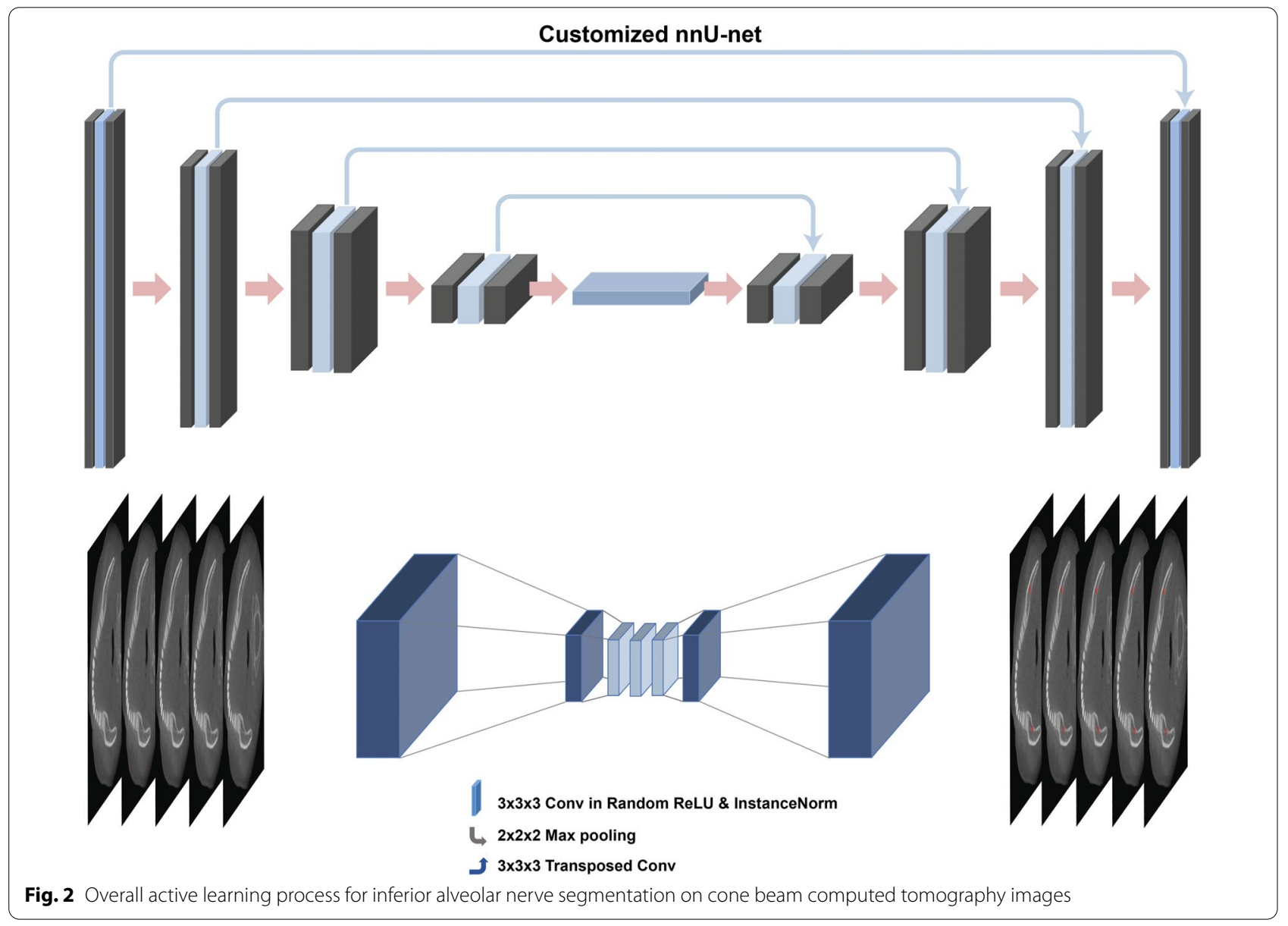


where $V_{\mathrm{gs}}$ was the volume for the ground truth, and $V_{\text {seg }}$ was the segmented volume.

In addition, the time required for segmentation of each dataset was measured at each training step. The time required for the training step was automatically measured and recorded on AVIEW Modeler software (version 1.0.3, Coreline Software, Seoul, Korea). Furthermore, a comparative evaluation of the accuracy between the manual and active learning segmentation obtained for the internal and external data was performed. For visual scoring, because intuitive scoring of IAN is difficult with 2D images, 3D scoring was performed using the AVIEW modeler software. The visual scoring evaluation was performed considering a grading scale, where four points were assigned for very accurate cases and one point for inaccurate cases. For visual scoring, three dentists conducted grade examination (manual ground truth $v s$. deep learning). The mean grade was calculated by the three dentists as follows. Grade 4 (Very accurate): when the labelled IAN completely matches the original IAN (over 95\%); Grade 3 (Accurate): when the labelled IAN almost completely matches the original IAN (85\%-9585-95\%); Grade 2 (Mostly accurate): when the labelled IAN depicts the site of the original IAN (over 50\%); Grade 1 (Inaccurate): when the labelled IAN depicts outside of the IAN or only matches a small area of the original IAN (under $50 \%)$.

\section{Results}

In this study, we validated the effect of active learning using a customized nnU-Net. The segmentation results improved as the steps progressed. The average DSC value for the IAN segmentation gradually increased after each learning step, with values of $0.48 \pm 0.11,0.50 \pm 0.11$, and $0.58 \pm 0.08$ (Mean $\pm \mathrm{SD}$ ), respectively, and showed the best results in the last step (Table 2). When accuracy was evaluated using an external dataset after learning, the DSC value for data obtained from B was $0.55 \pm 0.11$, and that for the data from $\mathrm{C}$ was $0.43 \pm 0.13$ (Table 3). The low-performing and high-performing IAN segmentation results for data obtained from $\mathrm{A}, \mathrm{B}$, and $\mathrm{C}$ were depicted (Fig. 3).

The average time required for segmentation of each dataset was $124.8 \mathrm{~s}$ for manual partitioning in the first

Table 2 Dice similarity coefficients after automatic segmentation of inferior alveolar nerve at the first, second, and last steps for the internal dataset (83 cases)

\begin{tabular}{llll}
\hline Mean \pm SD (range) & First step & Second step & Last step \\
\hline DSC & $0.48 \pm 0.11$ & $0.50 \pm 0.11$ & $0.58 \pm 0.08$ \\
& $(0.26-0.62)$ & $(0.32-0.62)$ & $(0.39-0.65)$
\end{tabular}

DSC, dice similarity coefficient; SD, standard deviation
Table 3 Dice similarity coefficients after automatic segmentation of inferior alveolar nerve in the test dataset [Internal: 15 cases (A), External: 20 cases each (B and C)]

\begin{tabular}{llll}
\hline Mean \pm SD (range) & Last step (A) & Last step (B) & Last step (C) \\
\hline DSC & $0.58 \pm 0.08$ & $0.55 \pm 0.10$ & $0.43 \pm 0.13$ \\
& $(0.39-0.65)$ & $(0.39-0.69)$ & $(0.0-0.65)$ \\
\hline
\end{tabular}

DSC, dice similarity coefficient; A, Korea University Anam Hospital; B, Korea University Ansan Hospital; C, Korea University Guro Hospital; SD, standard deviation

step and 143.4 s for manual correction after $\mathrm{CNN}$-assistance in the second step. In the last step, after CNNassistance, the time required for segmentation improved to $86.4 \mathrm{~s}$ after manual modification (Table 4). Further, in comparison to the first manual segment and the second stage, the time required decreased by approximately $38.4 \mathrm{~s}$ and $57.0 \mathrm{~s}$, respectively.

The visual scoring results were presented in Table 5. It was observed that the accuracy of manual segmentation was higher than that of automatic segmentation.

\section{Discussion}

Determining the exact location of the IAN is an important step in planning and realizing the treatment of the oral and maxillofacial areas using tools such as extraction, dental implants, and orthognathic surgery [1]. In general, the position of the IAN is designated and labeled manually in each section of the CBCT image. However, there are some limitations in manual IAN labeling using CBCT. First, it is difficult to accurately measure density and because of noise in the images [15]. Second, since the IAN travels in various directions in 3D, it is difficult to intuitively determine the exact shape [16]. Thirdly, it is difficult to determine whether the shape or position of the nerve has been deformed due to adjacent teeth or lesions [17]. Finally, if the cortical layer of the mandibular canal surrounding the IAN is unclear from the image, it is difficult to determine the exact location [18]. Due to these limitations, manually labelling an image slice can be time-consuming.

Therefore, in this study, a step-wise customized nnUNet method that enabled active learning with limited data was implemented. AI-based convenient labeling of IANs increases training efficiency. In fact, the overall improvement in the performance was observed after gradual refinements of AI with deep learning. As each step was completed, the DSC increased, and the segmentation time gradually decreased; approximately two-thirds of segmentation time were accomplished comparable to the manual segmentation.

In addition, in comparison to other studies, a multicenter dataset was used. The fact that using datasets from various institutions was a unique feature of this 


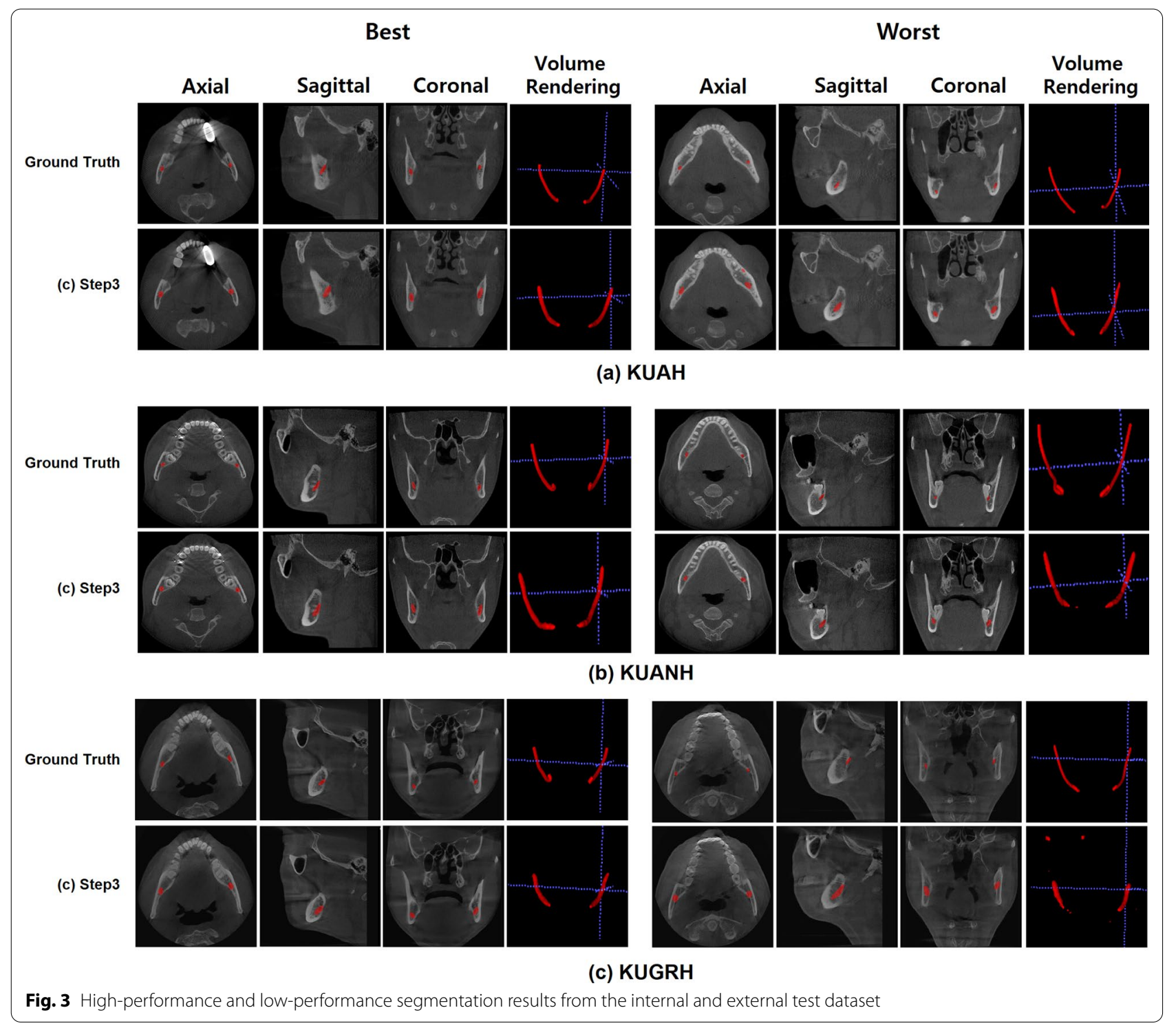

Table 4 Comparison of segmentation time for each dataset between the manual and convolutional neural network-assisted and manually modified segmentation approaches

\begin{tabular}{llll}
\hline & $\begin{array}{l}\text { First step } \\
\text { Manual } \\
\text { segmentation }\end{array}$ & $\begin{array}{l}\text { Second step } \\
\text { CNN-assisted } \\
\text { and manually } \\
\text { modified } \\
\text { segmentation }\end{array}$ & $\begin{array}{l}\text { Last step } \\
\text { CNN-assisted } \\
\text { and manually } \\
\text { modified } \\
\text { segmentation }\end{array}$ \\
\hline Average time (s) & $124.8 \pm 54.18$ & $143.4 \pm 102.66$ & $86.4 \pm 61.8$ \\
\hline
\end{tabular}

CNN, convolutional neural network

study that was different from other studies. The spectral structure of the trained data and the spectral structure of actual clinical data may be different, so external validation was mandatory in AI research [19]. It was demonstrated that AI performance can be improved through diversification of the dataset. Since the multicenter dataset was not utilized during learning, diversity in the AI learning stage could not be implemented. However, by comparing the segmentation efficiency of datasets acquired using equipment from different companies and specifications at different hospitals, it was possible to identify areas that need improvement through further study. Additionally, the consistency of the study model was confirmed with visual scoring of the results from each institution.

In this study, a customized nnU-Net method was used as the network. The nnU-Net method is derived from a 3D U-net and batch normalization [13]. 3D $\mathrm{U}-\mathrm{Net}$ is a widely used method for image segmentation, 
Table 5 Qualitative results from visual scoring of automatic inferior alveolar nerve segmentation on cone beam computed tomography from 53 randomly selected data (Internal: A, External: B and C)

\begin{tabular}{|c|c|c|c|c|c|c|c|c|c|c|c|c|}
\hline \multirow[t]{3}{*}{ Grade } & \multicolumn{6}{|c|}{ Manual } & \multicolumn{6}{|c|}{$\begin{array}{l}\text { nnU-net } \\
\text { (Last step of active learning) }\end{array}$} \\
\hline & \multicolumn{2}{|l|}{ A } & \multicolumn{2}{|l|}{ B } & \multicolumn{2}{|l|}{$\mathrm{C}$} & \multicolumn{2}{|l|}{ A } & \multicolumn{2}{|l|}{ B } & \multicolumn{2}{|l|}{ C } \\
\hline & Rt & Lt & $\mathbf{R t}$ & Lt & Rt & Lt & Rt & Lt & Rt & Lt & $\overline{\mathrm{Rt}}$ & Lt \\
\hline 4 (very accurate) & 14.3 & 14.3 & 19 & 19 & 19 & 19 & 11 & 9.7 & 10.7 & 12.3 & 5.3 & 4.7 \\
\hline 3 (accurate) & 0.7 & 0.7 & 0 & 0 & 0 & 0 & 2.7 & 3 & 7.3 & 5 & 8.7 & 7 \\
\hline 2 (mostly accurate) & 0 & 0 & 0 & 0 & 0 & 0 & 1.3 & 2 & 1 & 1.4 & 3.3 & 6.3 \\
\hline 1 (inaccurate) & 0 & 0 & 0 & 0 & 0 & 0 & 0 & 0.3 & 0 & 0.3 & 1.7 & 1 \\
\hline
\end{tabular}

A, Korea University Anam Hospital; B, Korea University Ansan Hospital; C, Korea University Guro Hospital; Rt, right; Lt, left

but its overall performance may be affected by $\mathrm{CNN}$ structure, pre-processing, and learning [20]. In contrast, nnU-Net is based on 3D U-Net, but it is more robust and shows more stable and improved performance owing to the self-adapting framework [13]. In the SegNet method, the decoder upsamples the functional map using the maximum pooling index instead of learning similar to that in a fully convolutional network (FCN) [21]. In contrast, the U-net up sampling operation learns to deconvolute the input function map, outputs it to the decoder, and combines it with the high-resolution function map [22]. In addition, as an extension of the 2D layer, a 3D layer was trained by randomly selecting $643 \mathrm{D}$ patches with $320 \times 320$ pixel images. The 3D network has the advantage of maintaining valid padding with the original $3 \mathrm{D}$ architecture, as it obtains more contextual information [23].

Previous studies on IAN segmentation with deep learning have provided considerable insights. Kwak et al. achieved an accuracy of about $0.82-0.99$ in IAN segmentation using $102 \mathrm{CBCT}$ datasets, 2D SegNet, and 2D and 3D U-Nets [24]. Jaskari et al. observed DSC values of about 0.57 to 0.58 using 637 CBCT datasets and 3D U-Nets [25]. Vinayahalingam et al. used 82 panoramic images and 2D U-Net and observed a DSC value of approximately $0.77-0.80$ [26]. Overall, our model performance was similar to that of previous AI models in literature.

In an author's previous study about automatic segmentation of the lesion and air of the maxillary sinus, which was conducted in the same way as this study, the DSC of the lesion of the maxillary sinus obtained through learning was $\sim 0.760$, and that of the air was $\sim 0.930$, while the segmentation time was $\sim 362 \mathrm{~s}$. Thus, in comparison to the manual segmentation a higher DSC and faster segmentation were achieved [14]. For IAN segmentation conducted in this study, a DSC of $\sim 0.58$ and a segmentation time of $\sim 86.4 \mathrm{~s}$ were observed, which were not as high as the maxillary sinus segmentation data. Presumably, in the maxillary sinus image, the difference in the Hounsfield unit (HU) inside and outside the segmentation area was prominent, resulting in a lower error value during segmentation. However, considering the IAN, the difference in $\mathrm{HU}$ between the inner and outer bone marrow in the mandibular canal is relatively small, which can result in errors during segmentation. Indeed, according to the literature, the $\mathrm{HU}$ in the mandibular canal is -726.4, and the $\mathrm{HU}$ in the cancellous bone is 416.2 [27]. Additionally, during IAN segmentation, a shorter time was observed. However, we need to consider a larger number of image cuts for comparison.

Another limitation of this study is that the shape of the anterior loop, which is partly inside the mental foramen at the end of the IAN, was not well implemented in deep learning (Fig. 4). We presume that due to the incomplete manual segmentation input data for learning, our model accuracy was lower. Thus, improvements in this direction should improve the accuracy of automatic IAN segmentation.

For the model developed in this study, we believe that effective learning is necessary to overcome the difference in segmentation efficiency for each anatomical application site. In addition, the initial input value i.e., the manual segmentation dataset, should be more precise to improve the accuracy. Moreover, the amount and diversity of the training dataset should be increased, and the segmentation performance should be improved using more diverse networks during training. Furthermore, additional multi-center datasets and comparisons with other segmented networks, such as cascade networks, must be performed to further verify the efficiency and stability of this methodology.

Manual IAN labeling is unintuitive, exhausting, and inconsistent, and it may not always be precise or consistent. Labeling using active learning in future will surely replace existing methods. Therefore, the active 

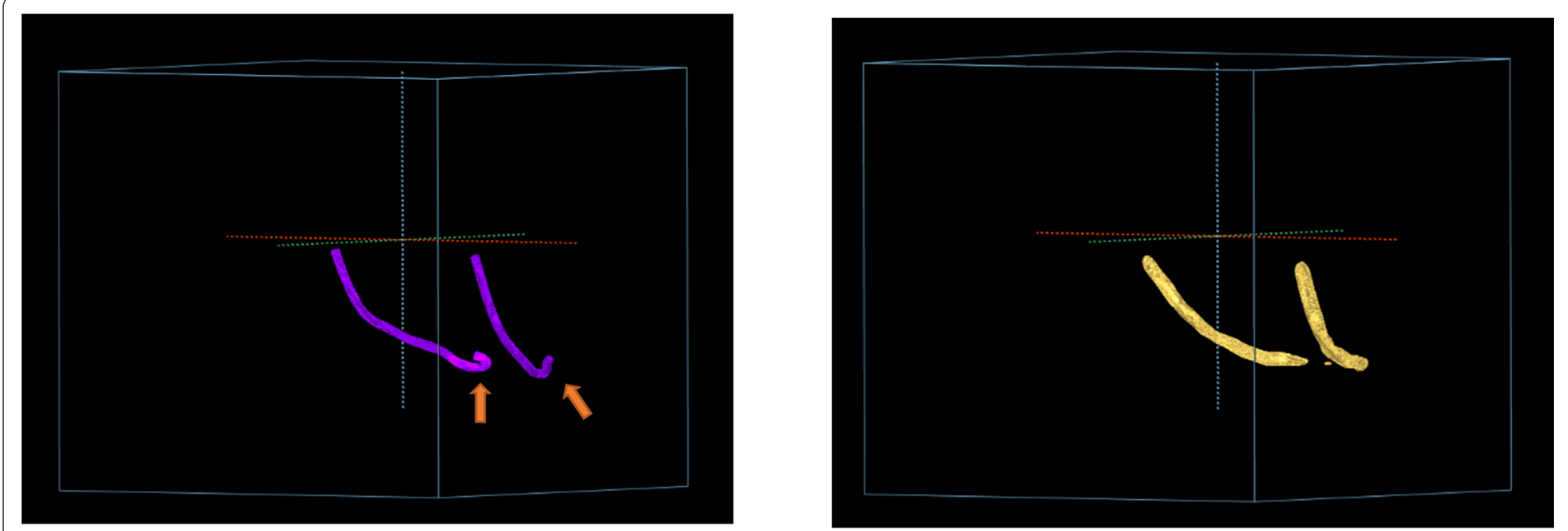

(a)

(b)

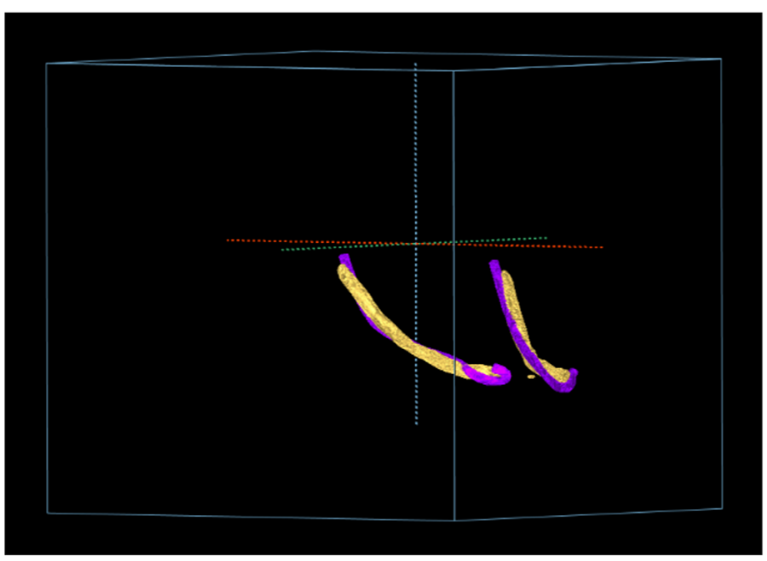

(c)

Fig. 4 Improperly implemented anterior loop shape of the inferior alveolar nerve during deep learning

learning framework presented in this study is a robust tool that can support manual labeling by improving the accuracy and reducing the procedural time.

\section{Conclusion}

Through this study, we confirmed that the deep active learning framework can improve the labeling accuracy of the IAN. Further, efficient learning on the limited CBCT dataset reduces the segmentation time, which can be implemented as a convenient automatic IAN labeling technique for future clinical use.

\section{Abbreviations}

2D: Two-dimensional; 3D: Three-dimensional; Al: Artificial intelligence; CBCT: Cone-beam computed tomography; CNN: Convolutional neural network; DSC Dice similarity coefficient; HU: Hounsfield unit; IAN: Inferior alveolar nerve; A: Korea University Anam Hospital; B: Korea University Ansan Hospital; C: Korea University Guro Hospital; ReLU: Random rectified linear unit; SSM: Statistical shape model.

\section{Acknowledgements}

We would like to thank Coreline Company and Ji-won Lee for software and technical support.

\section{Authors' contributions}

Conceptualization, S-KJ and H-KL; methodology, YC and I-SS; software, YC and SL; validation, S-KJ; formal analysis, H-KL and SL; investigation, S-KJ; resources, I-SS and SL; data curation, YC; writing —original draft preparation, S-KJ and $\mathrm{H}-\mathrm{KL}$; writing - - review and editing, I-SS and YC; visualization, S-HK; supervision, H-KL; project administration, I-SS; funding acquisition, I-SS and YC.

\section{Funding}

This work was supported by the Korea Medical Device Development Fund grant funded by the Korea government (the Ministry of Science and ICT, the Ministry of Trade, Industry and Energy, the Ministry of Health \& Welfare, the Ministry of Food and Drug Safety) (Project Number: 1711139098, KMDF_PR_20210527_0002).

\section{Availability of data and materials}

The datasets generated and/or analysed during the current study are not publicly available due [REASON WHY DATA ARE NOT PUBLIC] but are available from the corresponding author on reasonable request. 


\section{Declarations}

\section{Ethics approval and consent to participate}

This study was conducted in accordance with the Declaration of Helsink under the approval of the institutional ethics committee of each hospital [approved by the Institutional Review Board of A (2020AN0410, 11/01/2021), B (2021AS0041, 09/02/2021), and C (2021GR0148, 07/04/2021)].

\section{Consent for publication}

Not applicable.

\section{Competing interests}

The authors declare that they have no known competing financial interests or personal relationships that could have influenced the work reported in this paper.

\section{Author details}

'Department of Oral and Maxillofacial Surgery, Korea University Guro Hospital, 148, Gurodong-ro, Guro-gu, Seoul 08308, Republic of Korea. ${ }^{2}$ Department of Orthodontics, Korea University Guro Hospital, 148, Gurodong-ro, Guro-gu, Seoul 08308, Republic of Korea. ${ }^{3}$ Department of Medical Humanities, Korea University College of Medicine, 46, Gaeunsa 2-gil, Seongbuk-gu, Seoul 02842 Republic of Korea. ${ }^{4}$ Department of Radiology and Al Center, Korea University College of Medicine, Korea University Anam Hospital, 73, Goryeodae-ro, Seongbuk-gu, Seoul 02841, Republic of Korea. ${ }^{5}$ Department of Oral and Maxillofacial Surgery, Korea University Anam Hospital, 73, Goryeodae-ro, Seongbuk-gu, Seoul 02841, Republic of Korea.

Received: 6 October 2021 Accepted: 22 November 2021

Published online: 07 December 2021

\section{References}

1. Kim YT, Pang KM, Jung HJ, Kim SM, Kim MJ, Lee JH. Clinical outcome of conservative treatment of injured inferior alveolar nerve during dental implant placement. J Korean Assoc Oral Maxillofac Surg. 2013;39(3):127-33.

2. Lee $\mathrm{CH}$, Lee BS, Choi BJ, Lee JW, Ohe JY, Yoo HY, Kwon YD. Recovery of inferior alveolar nerve injury after bilateral sagittal split ramus osteotomy (BSSRO): a retrospective study. Maxillofac Plast Reconstr Surg. 2016;38(1):25

3. Xu GZ, Yang C, Fan XD, Yu CQ, Cai XY, Wang Y, He D. Anatomic relationship between impacted third mandibular molar and the mandibular canal as the risk factor of inferior alveolar nerve injury. $\mathrm{Br} J$ Oral Maxillofac Surg. 2013;51(8):e215-9.

4. Lee B, Park Y, Ahn J, Chun J, Park S, Kim M, Jo Y, Ahn S, Kim B, Choi S. Assessment of the proximity between the mandibular third molar and inferior alveolar canal using preoperative 3D-CT to prevent inferior alveolar nerve damage. Maxillofac Plast Reconstr Surg. 2015:37(1):30.

5. Widmer G, Horn W, Nagele B. Automatic knowledge base refinement: learning from examples and deep knowledge in rheumatology. Artif Intell Med. 1993;5(3):225-43.

6. Aggarwal R, Sounderajah V, Martin G, Ting DSW, Karthikesalingam A, King D, Ashrafian H, Darzi A. Diagnostic accuracy of deep learning in medical imaging: a systematic review and meta-analysis. NPJ Digit Med. 2021;4(1):65.

7. Yang R, Yu Y. Artificial convolutional neural network in object detection and semantic segmentation for medical imaging analysis. Front Oncol. 2021;11:638182

8. Hwang JJ, Jung $\mathrm{YH}$, Cho BH, Heo MS. An overview of deep learning in the field of dentistry. Imaging Sci Dent. 2019;49(1):1-7.

9. Kim J, Lee HS, Song IS, Jung KH. DeNTNet: Deep Neural Transfer Network for the detection of periodontal bone loss using panoramic dental radiographs. Sci Rep. 2019;9(1):17615.
10. Abdolali F, Zoroofi RA, Abdolali M, Yokota F, Otake Y, Sato Y. Automatic segmentation of mandibular canal in cone beam CT images using conditional statistical shape model and fast marching. Int J Comput Assist Rad. 2017;12(4):581-93.

11. Moris B, Claesen L, Sun $Y$, Politis C. Automated tracking of the mandibular canal in cbct images using matching and multiple hypotheses methods. In: Fourth international conference on communications and electronics (ICCE). 2012. p. 327-32.

12. Razi T, Emamverdizadeh P, Nilavar N, Razi S. Comparison of the Hounsfield unit in CT scan with the gray level in cone-beam CT. J Dent Res Dent Clin Dent Prospects. 2019;13(3):177-82.

13. Isensee F, Jaeger PF, KohI SAA, Petersen J, Maier-Hein KH. nnU-Net: a self-configuring method for deep learning-based biomedical image segmentation. Nat Methods. 2021;18(2):203-11.

14. Jung SK, Lim HK, Lee S, Cho Y, Song IS. Deep active learning for automatic segmentation of maxillary sinus lesions using a convolutional neural network. Diagnostics (Basel). 2021;11(4):688.

15. Pauwels R, Jacobs R, Singer SR, Mupparapu M. CBCT-based bone quality assessment: are Hounsfield units applicable? Dentomaxillofac Radiol. 2015:44(1):20140238

16. Kim ST, Hu KS, Song WC, Kang MK, Park HD, Kim HJ. Location of the mandibular canal and the topography of its neurovascular structures. J Craniofac Surg. 2009;20(3):936-9.

17. de Castro MAA, Barra SG, Vich MOL, Abreu MHG, Mesquita RA. Mandibular canal branching assessed with cone beam computed tomography. Radiol Med. 2018;123(8):601-8.

18. Oliveira-Santos C, Capelozza AL, Dezzoti MS, Fischer CM, Poleti ML, Rubira-Bullen IR. Visibility of the mandibular canal on CBCT cross-sectional images. J Appl Oral Sci. 2011;19(3):240-3.

19. Baldwin DR, Gustafson J, Pickup L, Arteta C, Novotny P, Declerck J, Kadir T, Figueiras C, Sterba A, Exell A, et al. External validation of a convolutional neural network artificial intelligence tool to predict malignancy in pulmonary nodules. Thorax. 2020;75(4):306-12.

20. Park J, Yun J, Kim N, Park B, Cho Y, Park HJ, Song M, Lee M, Seo JB. Fully automated lung lobe segmentation in volumetric chest $C T$ with 3D U-Net: validation with intra- and extra-datasets. J Digit Imaging. 2020;33(1):221-30.

21. Badrinarayanan V, Kendall A, Cipolla R. SegNet: a deep convolutional encoder-decoder architecture for image segmentation. IEEE Trans Pattern Anal Mach Intell. 2017;39(12):2481-95.

22. Norman B, Pedoia V, Majumdar S. Use of 2D U-Net convolutional neural networks for automated cartilage and meniscus segmentation of knee MR imaging data to determine relaxometry and morphometry. Radiology. 2018;288(1):177-85.

23. Gonzalez Sanchez JC, Magnusson M, Sandborg M, Carlsson Tedgren A, Malusek A. Segmentation of bones in medical dual-energy computed tomography volumes using the 3D U-Net. Phys Med. 2020;69:241-7.

24. Kwak GH, Kwak EJ, Song JM, Park HR, Jung YH, Cho BH, Hui P, Hwang JJ. Automatic mandibular canal detection using a deep convolutional neural network. Sci Rep UK. 2020;10(1):1-8

25. Jaskari J, Sahlsten J, Jarnstedt J, Mehtonen H, Karhu K, Sundqvist O, Hietanen A, Varjonen V, Mattila V, Kaski K. Deep learning method for mandibular canal segmentation in dental cone beam computed tomography volumes. Sci Rep. 2020;10(1):5842.

26. Vinayahalingam S, Xi T, Berge S, Maal T, de Jong G. Automated detection of third molars and mandibular nerve by deep learning. Sci Rep. 2019;9(1):9007.

27. Sande A, Ramdurg P. Comparison of hounsfield unit of CT with grey scale value of CBCT for hypo and hyperdense structure. Eur J Mol Clin Med. 2020:7(3):4654-8.

\section{Publisher's Note}

Springer Nature remains neutral with regard to jurisdictional claims in published maps and institutional affiliations. 